\title{
Políticas y logros en Tecnologías de Información y Comunicación en Venezuela
}

\author{
Genatios, Carlos* \\ Lafuente, Marianela** \\ * Profesor Titular del Instituto de Materiales y Modelos Estructurales UCV. Ex-Ministro de \\ Ciencia y Tecnología (1999-2002). Profesor de Postgrado UCV, Consultor Internacional. \\ E-mail: cgenatios@reaccion.ve \\ ** Profesora Titular del Instituto de Materiales y Modelos Estructurales UCV. Ing. Civil \\ UCV (1980). Profesora de Postgrado UCV, Consultor Internacional.
}

\section{Resumen}

El uso y difusión de las tecnologías de Información y Comunicación (TIC) es hoy en día un lugar común en las políticas públicas en la casi totalidad de los países del mundo. Venezuela carecía, hasta el final de la década del '90, de políticas de este tipo, tan sólo contaba, en algunos casos, con iniciativas aisladas. A partir de la creación del Ministerio de Ciencia y Tecnología (MCT), en 1999, se comenzaron a implementar políticas coherentes, las cuales dieron resultados de impacto inmediato. El objetivo de este trabajo es explorar a través del análisis documental los avances en los aspectos fundamentales que componen la plataforma actual de Venezuela en el desarrollo de las TIC, en particular en lo relativo a la conectividad, la capacitación, los contenidos y la economía digital. Se destacan como logros principales, el crecimiento más acelerado de usuarios de Internet en América Latina, así como generar el crecimiento más acelerado de economía digital en la región. Se resaltan los elementos más importantes en la formulación de las políticas públicas en el área, y se subraya la necesidad de dar continuidad a estos esfuerzos, a fin de no perder el alcance y las perspectivas logradas. Se concluye que es vital mantener un esfuerzo sostenido en las áreas de capacitación, conectividad, contenidos, gobierno electrónico, economía digital, los cuales deben continuar siendo liderizados vigorosa y entusiastamente por el gobierno nacional, y de acuerdo a la Ley Orgánica de Ciencia, Tecnología e Innovación, el rol recae sobre el Ministerio de Ciencia y Tecnología.

Palabras clave: Ministerio de Ciencia y Tecnología, tecnología de información y comunicación, políticas públicas. 


\title{
Policy and Advances in I nformation and Communication Technology in Venezuela
}

\begin{abstract}
The use and diffusion of Information and Communication Technology (ICT) is nowadays a common area of public policy in almost every country in the world. Venezuela lacked, until the end of the 1990s, policies of this type which only existed in a few isolated cases. The creation of the Ministry of Science and Technology (MST), in 1999, opened the door to the implementation of coherent policies which caused immediate impact. The objective of this paper is to explore, through documentary analysis, advances in the fundamental aspects that make up this platform in Venezuela and the development of ICTs especially those related to connections, capacity, contents and digital economy. The major advances noted are the accelerated increase in the number of Internet users in Latin America as well as the generation of accelerated growth in the digital economy of the region. The most important elements that emerge in the formulation of public policy in this area are explained, and the need for continuity in these efforts is underlined in order not to lose the perspectives and growth already achieved. The conclusion is that it is vital to maintain this effort in the areas of capacity development, connectivity, contents, electronic government, and digital economy, all of which should be vigorously and enthusiastically headed by the national government. According to the organic law related to science, technology and innovation, the principal role falls on the Ministry of Science and Technology.
\end{abstract}

Key words: Ministry of Science and Technology, informational and communicational technology, public policy.

Recibido: 02-09-27. Aceptado: 02-12-05

\section{I ntroducción}

En la era de la sociedad del conocimiento, la información y el conocimiento son factores claves en los procesos de producción y creación de riqueza. En este sentido, las tecnologías de información y comunicación (TIC) cumplen un papel determinante, por lo que los ciudadanos en general y los gobiernos en especial, de aquellas naciones que van a la vanguardia del desarrollo, han comprendido su lógica intrínseca, la cual impulsan, y han tratado de organizar estas tecnologías para incrementar su productividad y bienestar. Pero, en sentido prioritario, las han visualizado como un instrumento que contribuye al 
logro de amplios objetivos nacionales, tanto sociales como económicos, y principalmente como soporte a las políticas nacionales y programas de desarrollo.

En efecto, la experiencia de las organizaciones y las naciones contemporáneas que han tenido éxito en alcanzar sus misiones fundamentales, indica que en cada caso el uso apropiado de las TIC ha sido un factor determinante en el fortalecimiento de la capacidad de enfrentar los problemas que surgen de la complejidad del mundo actual. En este empeño, el resultado de este proceso ha sido que estas tecnologías emergen como instrumentos transversales a la sociedad, es decir, penetran y se integran prácticamente en todas las actividades y sectores de la misma, y hoy no es posible prescindir de ellas, puesto que en sí mismas pautan el tiempo, la manera de trabajar, aprender, comunicarse y de gobernar.

Sin embargo, las TIC no son una panacea para solucionar todos los problemas del desarrollo, tales como la pobreza. Por ello, las políticas dirigidas a su desarrollo deben ir acompañadas de estrategias que reduzcan la sustancial brecha entre los "que tienen" y "no tienen" conocimiento e información. Las desigualdades acumuladas en lo educativo, económico, social y político, hacen que las personas con una mejor educación, con altos ingresos económicos, con poder político y gran movilidad social posean un mayor acceso a los conocimientos y a la información, en comparación a aquellas personas que no han tenido las mejores oportunidades. Esta situación de desequilibrio es posible también detectarla en lo que respecta a las ciudades o regiones pobres y rurales.

Dentro de este contexto, se enfrentó en los dos últimos años, el desafío de impulsar iniciativas de carácter público y privado, concebidas a partir del consenso y motorizadas con el entusiasmo de una comprometida gestión pública, que permitieron que Venezuela diera un salto muy significativo en el avance hacia una sociedad del conocimiento y de la información, es decir, una sociedad dotada de habilidad, capacidad y pericia para generar y captar nuevos conocimientos y tener acceso a la información, a los datos y a los conocimientos, absorberlos y utilizarlos eficazmente con el apoyo de las TIC.

\section{Diagnóstico y crecimiento reciente}

Venezuela presenta hoy un adecuado potencial para el desarrollo de las Tecnologías de la Información, comparado con otros países de la región. Pasamos a enumerar los aspectos fundamentales de lo logros recientes, los cuales justifican esta afirmación.

Según encuesta de Datanálisis (2001), Venezuela tenía para el segundo semestre de 
1999, 207.000 usuarios de Internet. Para el primer semestre de 2000 esa cifra subió a 526.000, y para el segundo semestre de 2000 llegó a 817.000, lo cual representó un crecimiento del 300\%. Para el 2001, 1.200 .000 personas eran usuarios de Internet $(4.8 \%$ de la población). A pesar del importante crecimiento experimentado en ese período, Venezuela está por debajo de los niveles alcanzados en Chile (11.6\%), Uruguay (11.1\%), Argentina (10.38\%), Brasil (6.98\%), y Costa Rica (6.74\%). Sin embargo, las proyecciones de crecimiento del número de usuarios para Venezuela hasta el año 2005 son de 1167\%, las más altas de América Latina, por encima de México (877\%), Colombia (780\%), Argentina (775\%) y Brasil (402\%), de acuerdo a cifras de Jupiter Communications (2002).

Los usuarios venezolanos se distribuyen desigualmente, según el estrato social, indicando que los sectores más desfavorecidos tienen menos acceso a las nuevas tecnologías (más del $80 \%$ de los usuarios pertenecen a los estratos A, B y C de la población y ganan más de cuatro veces el salario mínimo del país), mientras que los estratos D y E ocupan el 14\%. La distribución de los usuarios es desigual desde un punto de vista geográfico: $71 \%$ de ellos se encuentran en el Distrito Capital, 5.7\% en Maracaibo y $3.5 \%$ en Valencia (Cavecom-e, 2001).

El programa más exitoso en la difusión de las TIC, y en el impulso de la conectividad fue sin duda el de los Infocentros, emprendido y financiado por el Ministerio de Ciencia y Tecnología en el año 2001 (MCT, 2001a-2001b). En ese programa se implantaron 243 centros de acceso gratuito para la población en más de doscientos municipios, en toda la geografía nacional, lo cual permite más de 7.5 millones de visitas al año. La ubicación de estos infocentros incluye lugares remotos de muy difícil acceso, como la selva amazónica, en barrios populares o en cárceles, orientándose a sectores de la población que por otras vías tendrían gran dificultad para lograr "conectarse" en la red; por lo tanto este programa impulsa la participación de los menos pudientes en el ciberespacio. Los infocentros son salas dotadas de computadores y acceso a Internet, por vía de cableado o inalámbrica, ubicadas, en su mayoría en instituciones públicas, principalmente en bibliotecas públicas, o en centros comunitarios, alcaldías, museos, entre otros.

La promulgación de la Ley de Firmas y Datos Electrónicos en 2001, es un elemento definitorio y de vanguardia de la condiciones de desarrollo de las TIC. La misma fue elaborada en un proceso de amplia participación del sector oficial, la academia y el sector privado. Esta ley tiene por objeto “otorgar y reconocer eficacia y valor jur ídico a la Firma Electrónica, al Mensaje de Datos y a toda información inteligible en formato electrónico, independientemente de su soporte material, atribuible a personas naturales o jur ídicas, 
públicas o privadas, así como regular todo lo relativo a los Proveedores de Servicios de Certificación y los Certificados Electrónicos" (Asamblea Nacional, 2001: Art. 1). Esta ley requiere actualmente de la culminación de su reglamento. En febrero de 2000 se dictó el decreto 825 sobre el uso del Internet como prioridad nacional, en el cual se impulsa al uso de las Tecnologías de la Información en las instituciones gubernamentales (Asamblea Nacional, 2000a).

El comercio electrónico en Venezuela es también un sector de crecimiento importante. Las proyecciones para el año 2005 nos colocan en primer lugar en América Latina en tasa de crecimiento, por encima de Argentina (7193\%), México (6068\%) y Chile (4357\%) (Júpiter Communications, 2001).

En relación con las capacidades de producción de Software, esta industria en Venezuela tiene una apreciable capacidad competitiva, con más de 300 empresas en este ramo. Además, se han abierto recientemente alrededor de 500 empresas de diseño y hospedaje de páginas Web y cerca de cincuenta empresas líderes mundiales en el campo de las $\mathrm{TI}$ tienen oficinas en Venezuela (CONAPRI).

Venezuela tiene uno de los más elevados índices de penetración de telefonía móvil celular de Latinoamérica -según datos de CONATEL, $26.2 \%$ en 2001 , con un crecimiento de $19.14 \%$ en 2000-2001-. La penetración de telefonía fija se estima en un $11.2 \%$ en 2001 y la televisión por suscripción en el país presenta uno de los índices de crecimiento más elevados de Latinoamérica, con una penetración de 18\% en 2001 (CONATEL, 2002).

El sector TIC contribuyó con un 3.4\% del PIB en 1999 (US\$ 4568 millones), de los cuales US\$2563 en telecomunicaciones (Banco Central de Venezuela, 2002). En el año 2000, el mercado de hardware fue de US\$850 millones, lo que representó un crecimiento del 25\% respecto a 1999. Para 2001 se estimó un aumento del 30\%. Las ventas de computadores personales registraron en 1999 un crecimiento del 30\% (CONAPRI, 2000). El mercado local de software es de US\$244 millones, con un crecimiento anual de ventas del $27 \%$ entre 1997 y 2000. Las empresas exportan, en promedio, el 19\% de sus ventas totales. Se calcula que la industria del software proporciona empleo a más de 4500 personas (CONAPRI, 2000). IBM estimó que la inversión de Hardware y Software en Venezuela en 2001 era de 1800 millones de US\$. Para 2002 lo estima de 1600 millones de US\$ (conversaci ón entre Ministro de MCT y presidente IBM Venezuela).

La manufactura de componentes electrónicos se limita en Venezuela al ensamblaje de computadores personales. Existen algunas empresas locales que producen, por ejemplo, 
casetas de teléfonos, bases de relés, pedestales para fibra óptica y cables coaxiales, protectores de teléfonos, plantas eléctricas, y ciertos equipos suplementarios, como reguladores de voltaje y estabilizadores de frecuencia (MCT y CONAPRI, 2001).

Venezuela ha sido uno de los países con mayor porcentaje de inversión extranjera en el área de telecomunicaciones. En el año 2001, el 84\% de la inversión extranjera en áreas no petroleras, fue en este sector: aproximadamente, 611 millones de US\$ (CONATEL, 2002).

Las empresas del sector de telecomunicaciones en Venezuela, se dedican principalmente a las áreas de servicios y al área comercial. Las actividades de investigación y desarrollo son casi nulas, y las casas matrices ubican sus unidades de I+D en el extranjero. Sin embargo, la ley de telecomunicaciones crea un fondo sectorial para la investigación y desarrollo en telecomunicaciones (FIDETEL), administrado por el Ministerio de Ciencia y Tecnología (MCT), alimentado a partir del 2001 con aportes del sector empresarial, $(0.5 \%$ de la inversión bruta del sector de telecomunicaciones) lo que debería contribuir, a corto plazo, a darle impulso a estas actividades y adelantar los procesos de creación, transferencia de tecnología e innovación en el país (Asamblea Nacional, 2000b). Iniciativas de los sectores privado y universitario, son apoyadas por FIDETEL .

Recientemente se ha logrado que más del 90 por ciento de los principales organismos de la administración pública tengan presencia en Internet, aun cuando en muchos casos es muy preliminar (MCT-CNTI, 2001 y CNTI, 2001). Se lanzó el servicio de declaración en línea para pagos de impuestos. Se han desarrollado servicios de información a través de páginas web de organismos como el Tribunal Supremo de Justicia y el Ministerio de Finanzas. Sin embargo, alcanzar el objetivo de un Gobierno en únea, es un proceso a mediano plazo, que implica la coordinación adecuada de los organismos públicos y la actualización y mantenimiento permanente de los contenidos y servicios.

Entre 2000-2002, el MCT, a través del Centro Nacional de Tecnologías de la Información ( $\mathrm{CNTI})$ desarrolló los siguientes portales:

www.minnovacion.gov.ve y www.miproyecto.gov.ve: recepción y evaluación de proyectos ante el MCT y FONACIT (70.000 visitas al año) (MCT, 2002a y 2002b)

www. venezuelaproductiva.gov.ve Servidor temático de Producción y Comercio para dar valor agregado a la información del sector productivo, y facilitar trámites (14.500 visitas mensuales) (MCT-CNTI, 2002a). 
www.venezuelasaludable.gov.ve Servidor Temático en salud (8.500 visitas mensuales). Información sobre necesidades y requerimientos de instituciones y empresas del sector y crea la base para servicios de Telemedicina (MCT-CNTI, 2002b).

www.venezuelainnovadora.gov.ve Servidor Temático de Ciencia y Tecnología: oferta y demanda de información en la materia, vincula actores del área, información sobre revistas científicas (MCT-CNTI, 2002c).

www.venezuelatemática.gov.ve Servidor Temático Bibliotecario: información clasificada de prensa (MCT-CNTI, 2002d).

www.pep.redpetroleo.edu.ve Red "El Petróleo es una Escuela" en colaboración con PDVSA: intercambio y desarrollo del conocimiento petrolero aplicado por el profesorado de educación básica (PDVSA, 2002).

La AGENDA TI-educación del MCT, desarrolló contenidos educativos vigentes en el país. Con 1.000 millones de bolívares, se financiaron proyectos desarrollados por empresas, centros de I+D y Universidades, y se consolidó la red RENA, de contenidos educativos en educación básica www.rena.edu12.ve (MCT-CNTI, 2002e).

Se desarrollaron portales en instancias de cooperación internacional. Como ejemplo, se pueden mencionar las redes Latinoamérica y el Caribe, Tecnologías de Información y Comunicación ( $\mathrm{AACTIC)}$, con contenidos de educación primaria, y Red Internacional Virtual en Educación (RIVED), con contenidos para el área de Ciencias en educación secundaria. La primera (LACTIC) es una iniciativa emprendida por el MCT de Venezuela a mediados de 2000, dentro del cuadro de la comisión Económica y Social de La Organización de las Naciones Unidas, y abarca actualmente a un número importante de países de la región latinoamericana y del Caribe. La segunda (RIVED), abarca actualmente a Argentina, Brasil, Colombia y Venezuela.

En cuanto a la formación del talento humano en Venezuela, se estima que en las áreas de informática, computación, sistemas, ingeniería eléctrica y electrónica, telecomunicaciones, electrotecnia, y otras relacionadas, existen actualmente, alrededor de 20.000 profesionales de nivel universitario. Se preparan, actualmente, unos 60.000 en 26 universidades y 48 institutos universitarios de tecnología nacionales y, en los próximos cinco años se espera contar con 32.000 adicionales. Debe resaltarse la alta calidad de los centros educativos existentes, en especial las universidades nacionales más importantes, 
lo cual es un significativo potencial para el desarrollo del sector. Desarrollan actividades de alto nivel en la formación de profesionales y posgraduados, así como en la creación de software para resolución de complejos problemas de ingeniería. A finales de 2001, el MCT firmó con IBM un convenio para formar 16.000 desarrolladores de software en cuatro años. En convenio firmado con IBM, se creó en el 2001 una red nacional de centros de formación, asociada a los Institutos Tecnológicos Universitarios (IUT), donde se acreditarán especialistas en desarrollo de software y e-business, con posibilidades inmediatas de inserción en el mercado laboral nacional e internacional (MCT, 2001).

El MCT, por medio del establecimiento de convenios con importantes empresas internacionales con filiales en el país, como Microsoft, Cisco, Sun e IBM, diseñó, suscribió e inició en los años 2001 y 2002, programas destinados a formar técnicos en redes y desarrolladores de software, acreditados según estándares internacionales, además de haberse instalado una red nacional de academias de formación técnica, para formar capacidades en TIC y desarrollo de software, asociada a infocentros y centros universitarios en distintas regiones del país. Se desarrolló un sistema nacional de formación técnica en redes, para capacitar estudiantes en el diseño y mantenimiento de redes y generación de software.

EI MCT inició un gran esfuerzo en apoyar la creación de empresas en el sector, así como en la modernización de pequeñas y medianas empresas a nivel nacional, mediante los programas de desarrollo y aplicación de TI del MCT. Estos programas estimulan la incorporación de componentes tecnológicos en el sector productivo (MCT, 2001).

Todo este avance ha hecho que Venezuela pase, en el índice Mundial de la Sociedad de la Información, de ocupar la posición número 44 en agosto de 2000 a la posición número 37 en julio de 2001 (CONAPRI, 2002).

\section{Conclusiones: Perspectivas en políticas públicas}

Lo resumido de este espacio limita un diagnóstico más exhaustivo. Sin embargo, debemos incluir algunos elementos que contribuyan a la discusión sobre las perspectivas actuales.

Es vital mantener un esfuerzo sostenido en las áreas de capacitación, conectividad, contenidos, gobierno electrónico y economía digital, los cuales deben continuar siendo liderizados vigorosa y entusiastamente por el gobierno nacional, y de acuerdo a la Ley Orgánica de Ciencia, Tecnología e Innovación, el rol rector recae sobre el MCT. 
Para continuar con estos esfuerzos deben convocarse insistentemente a los diversos actores a la conformación de agendas a nivel nacional, que deben continuar siendo de los sectores académico y empresarial, y la presencia activa del sector gobierno es vital, ya que la definición de sus requerimientos establece la demanda principal para el desarrollo de las TIC y de la economía asociada. Debe profundizarse el esfuerzo para la definición de programas de desarrollo regional y local a nivel estatal y municipal, y para ello es necesario insistir en la capacitación y definición de planes en las distintas instancias públicas. Los talleres de fortalecimiento de la gestión regional iniciados por el MCT el año (2001) son una estrategia adecuada para tal fin, debido a que estimulan la formulación de planes de desarrollo a nivel local, articulándolos con las políticas a nivel nacional, y mejoran las capacidades de gestión local, contribuyendo a la participación y a la gobernabilidad. Este esfuerzo es difícil, pero de vital importancia, a fin de aprovechar las oportunidades que las TIC presentan para el desarrollo del país. Con el propósito de aprovechar las ventajas que las TIC ofrecen, tenemos como primera prioridad la capacitación del usuario, el cual en muchos casos es el mismo gobierno, para que así pueda conocer sus necesidades, establecer sus demandas y llegar a acuerdos con el sector productivo, tanto académico como empresarial.

Debemos fortalecer la demanda capacitando al usuario, así como al recurso humano que pueda elaborar productos ajustados a las necesidades nacionales e internacionales, y que estimulen el intercambio especialmente a nivel latinoamericano, a fin de aprovechar a la numerosa comunidad hispanoparlante.

A fin de fortalecer la oferta es vital estimular la industria nacional de Software, continuando los programas de incubadoras de empresas, de innovación del sector productivo y la conformación y utilización de capitales de riesgo.

Es inquietante que el sector privado, de acuerdo con opiniones aparecidas en la prensa y en distintos foros, vea con preocupación que estas exitosas iniciativas ejecutadas en el período 2000-2001 se hayan visto casi detenidas en el 2002. La continuidad de las políticas públicas y de los programas iniciados, es una condición indispensable para lograr el impacto esperado en este importante sector de desarrollo y consolidar una industria nacional de software, aprovechando nuestras ventajas y potencialidades actuales, que pueden ubicar a Venezuela, competitivamente, a la cabeza de la región en esta área.

Estos elementos deben seguir siendo, sin duda, prioridades de las políticas públicas en TIC. 


\section{Bibliografía Citada}

1. Asamblea Nacional (2001), Ley de Mensajes de Datos y Firmas Electrónicas, Gaceta Oficial No. 37202, del 28 de febrero. Caracas, Venezuela.

2. Asamblea Nacional (2000a), Decreto No. 825, Gaceta Oficial No. 36.955, del 22 de mayo. Caracas, Venezuela.

3. Asamblea Nacional (2000b), Ley Orgánica de Telecomunicaciones, Gaceta Oficial No 36.970 del 12 de junio. Caracas, Venezuela.

4. Banco Central de Venezuela (2002), Sección de información estadística http:// www.bcv.org.ve, Fecha de Consulta 23/09/2002.

5. Cámara Venezolana de Comercio Electrónico (CAVECOM-e) (2002), Sección de indicadores http:// www. cavecom-e. org.ve, Fecha de consulta 26/7/2002.

6. CNTI (2001), Plan Nacional de Tecnologías de Información, http://www.cnti.ve, Fecha de Consulta 24/04/2002.

7. Comisión Nacional de Telecomunicaciones (CONATEL) (2002), Sección de indicadores http:// www.conatel.gov.ve, Fecha de Consulta 20/09/2002.

8. Datanálisis (2002), Resultados de encuestas 2001, sección noticias, http://www. cnti.ve. Fecha de consulta 24/7/2002.

9. Jupiter Communications (2002), Resultados de encuestas, sección noticias, http:// www.cnti.ve, Fecha de consulta 24/7/2002.

10. MCT (2001a). Portal de recepción y evaluación de proyectos, http://www.minnovacion.gov.ve, Fecha de Consulta 28/10/2002.

11. MCT (2001b). Portal de recepción y evaluación de proyectos, http:// www.miproyecto.gov.ve, Fecha de Consulta 28/10/2002.

12. MCT-CNTI (2002a), Servidor temático de producción y comercio, http:// www.venezuelaproductiva.gov.ve, Fecha de Consulta 28/10/2002. 
13. MCT-CNTI (2002b), Servidor temático en salud, http:// www.venezuelasaludable.gov.ve, Fecha de Consulta 28/10/2002.

14. MCT-CNTI (2002c), Servidor temático en Ciencia y Tecnología, sector bibliográfico, http:// www.venezuelainnovadora.gov.ve, Fecha de Consulta 28/10/2002.

15. MCT-CNTI (2002d), Servidor temático bibliotecario, http:// www.venezuelatemática.gov.ve, Fecha de Conuslta 28/10/2002.

16. MCT-CNTI (2002e), Contenidos de educación básica, http://www.rena.edu12.ve, Fecha de Consulta 28/10/2002

17. MCT-CNTI (2001), Infocentros, http:// www.infocentros.gov.ve, Fecha de Consulta 21/08/2002. Caracas, Venezuela.

18. MCT- CONAPRI (2001), Folleto para la promoción de la inversión en Venezuela, http:// www.conapri.org, Fecha de Consulta 2001.

19. PDVSA (2002), Red "El petróleo es una Escuela", http:// www.pep.redpetroleo.edu.ve, Fecha de Consulta 28/10/2002. 